\title{
Le rôle du récit de vie dans le discours politique de
} Lula

The role of Lula's Life Story in his Political Speeches

Ida Lucia Machado

\section{OpenEdition}

1 Journals

Édition électronique

URL : http://journals.openedition.org/aad/1166

DOI : $10.4000 /$ aad. 1166

ISSN : $1565-8961$

Éditeur

Université de Tel-Aviv

Référence électronique

Ida Lucia Machado, "Le rôle du récit de vie dans le discours politique de Lula ", Argumentation et Analyse du Discours [En ligne], 7 | 2011, mis en ligne le 15 octobre 2011, consulté le 23 septembre 2019. URL : http://journals.openedition.org/aad/1166 ; DOI : 10.4000/aad.1166

Ce document a été généré automatiquement le 23 septembre 2019.

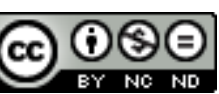

Argumentation \& analyse du discours est mis à disposition selon les termes de la licence Creative Commons Attribution - Pas d'Utilisation Commerciale - Pas de Modification 4.0 International. 


\title{
Le rôle du récit de vie dans le discours politique de Lula
}

The role of Lula's Life Story in his Political Speeches

\author{
Ida Lucia Machado
}

\section{Présentation de la problématique}

1 Le récit de vie est un genre introduit en France il y a presque une quarantaine d'années, par un chercheur en sciences sociales, Daniel Bertaux (1976). Au Brésil, il est utilisé surtout dans le domaine de l'histoire, qui a adopté le syntagme "Histoire de vie", traduction littérale de l'américain life story. La prise en compte de ce genre en analyse du discours (AD) est relativement récente, bien que Boyer ait, dès la fin des années 1980 et en se référant à Adam (1984) et Van Dijk (1983), utilisé le terme dans une perspective textuelle en le définissant comme « un discours narratif autobiographique, c'est-à-dire comme un certain type d'organisation textuelle» (1988: 55). Plus récemment, vers 2005, le regain d'intérêt manifesté envers l'autobiographie dans une perspective d'AD a placé les recherches sur le récit de vie sur le devant de la scène.

2 Cet article propose une réflexion non sur le genre du récit de vie en soi, mais bien plutôt sur son rôle dans les discours politiques de Luis Ignacio Lula da Silva, plus simplement connu sous le nom de Lula, qui a été président du Brésil de 2003 à $2010^{1}$. Il s'agit d'analyser quelques-uns de ses dits qui mêlent expressément des souvenirs de sa vie passée aux discours politiques d'actualité, ou encore qui font allusion à sa vie de façon plus ou moins directe. En ce sens, le récit de vie comprend une dimension argumentative qui permet de construire un ethos présidentiel par le jeu qu'il instaure entre l'individuel et le collectif, créant ainsi un effet de pathos qui appelle à l'identification.

3 L'article est divisé en trois parties. La première offre quelques données de la vie de Lula, qui sont nécessaires pour bien le situer dans le contexte social et politique brésilien et préparer ainsi la seconde partie centrée sur le genre récit de vie; on y survolera les recherches déjà menées sur la question en France pour, ensuite, 
développer quelques réflexions sur le "pourquoi» de l'introduction du récit de vie dans le discours politique. On examinera la façon dont le récit de vie a contribué à la formation d'un ethos positif de Lula en tant qu'homme politique ; on verra comment, en conjuguant habilement passé, présent et futur dans ses discours, il a encouragé une réaction d'adhésion ou d'identification à son ethos de président. Une brève troisième partie met l'accent sur le rôle d'un personnage important et émouvant dans la vie de Lula: sa mère. Elle commande, d'une certaine façon, les souvenirs évoqués par le Président. Chacune de ces trois parties s'appuie sur la présentation et l'analyse d'extraits des discours de Lula.

4 On essaye enfin, dans la conclusion, de répondre aux questions que l'utilisation du récit de vie dans le discours d'un homme politique peut soulever: quel est le type de stratégie mobilisée par Lula ? Doit-il être considéré comme un simple moyen de captation d'auditoire, ou comme faisant partie inhérente d'un discours " populiste ", au sens que Charaudeau prête à ce terme ? Selon lui, le langage du leader populiste doit, entre autres, être « simple et percutant, prétendant (mais prétendant seulement) ne pas pratiquer la langue de bois, s'accompagnant d'une vocalité et d'une gestuelle simple et forte, jouant sur le 'je-nous' exclusif qui s'oppose aux autres » (Charaudeau $2008: 83$ ). Le langage de Lula présente certes quelques-unes de ces facettes, mais peut-on pour autant le qualifier de «populiste»?

5 Je voudrais préciser que l'article n'a aucune prétention d'exhaustivité sur les sujets mentionnés. Il entend porter un regard analytique sur la façon dont un genre (le récit de vie) s'insère dans un autre (l'allocution présidentielle comme forme de discours politique) et sur les possibles stratégies argumentatives qui soutiennent cet ensemble. Il espère ainsi poser les jalons d'une étude qu'il serait intéressant de prolonger.

\section{Brèves informations sur Lula, Président du Brésil de 2003 à 2010}

6 Lula n'a pas fréquenté de grandes écoles pour devenir politicien. Son apprentissage du discours communicatif et argumentatif a été fait in loco, c'est-à-dire sur les lieux mêmes de sa prestation en tant que syndicaliste, puis président du Syndicat des Métallurgistes à l'Etat de São Paulo, où il est venu vivre pour échapper à la famine et à la misère de sa ville natale, située au nord du Brésil, dans l'Etat du Pernambouc.

7 Avant d'être métallurgiste, Lula a quitté l'école très tôt pour travailler, en assumant plusieurs petits boulots dans la rue pour aider sa nombreuse famille à survivre dans la jungle urbaine. Il a ainsi été cireur de chaussures, vendeur de cacahuètes, messager d'entreprises, etc. A 12 ans, il a trouvé son premier vrai emploi, dans une blanchisserie. Mais c'est à l'âge de 14 ans qu'il a eu sa Carte de Travail signée par les Entrepôts Généraux Columbia. Ensuite, il a été transféré vers la Fabrique de vis Marte. Pendant trois ans, comme il arrive souvent pour une grande partie des Brésiliens, il a travaillé le jour et a suivi, le soir, des cours techniques (gratuits) au SENAI (Service national de l'Industrie). Ces cours lui ont permis d'obtenir le certificat d'ouvrier tourneur mécanicien et d'être embauché dans une des principales usines métallurgiques du pays, dans la ville de São Bernardo do Campo, qui fait partie de la région appelée « $A B C$ paulista ", l'un des plus importants pôles industriels du pays. Dans son travail, petit à petit, il s'est mis en contact avec des groupes et des mouvements syndicaux et est 
devenu premier secrétaire et puis président du syndicat, représentant (élu par ses pairs) d'à peu près cent mille travailleurs.

Curieusement, le futur dirigeant du Parti des Travailleurs n'a pas un passé de militant de gauche, car il ne s'est jamais inscrit dans un parti radicalement opposé au gouvernement militaire avant de fonder le sien propre. En d'autres termes, s'il songeait à plus de justice pour lui et pour ces collègues ouvriers, il ne s'intéressait pas trop au militantisme des partis politiques de la gauche brésilienne qui a, tant bien que mal, toujours continué son combat contre le pouvoir des dictateurs. Ce «détachement »a donné à Lula une certaine liberté d'action au sein de son syndicat et a fait de lui, aux yeux des patrons et entrepreneurs, une sorte de "syndicaliste à qui on peut faire confiance ", quelqu'un avec qui ont peut "discuter ", puisque il était plutôt perçu comme «neutre» du point de vue politique. Cependant, comme il l'avoue lui-même ${ }^{2}$, cette attitude a fini par changer au fil des ans. Ainsi, en tant que syndicaliste, il n'a pas hésité à encourager et à assumer des grèves, dont l'une des plus grandes du pays, celle de 1979, quand 170.000 métallurgistes ont réussi à paralyser complètement «l'ABC paulista».

De 1979 à 1982, Lula et ceux qui l'appuyaient ont réussi à créer un nouveau parti politique au Brésil, le Parti des Travailleurs, connu sous le sigle «PT». On peut se demander comment cela est devenu possible dans un pays gouverné par des dictateurs. Il faut donc rappeler qu'à partir de 1977, le pouvoir en place a mis en pratique, bien que très lentement, une sorte de "politique de transition » dont les buts évidents étaient avant tout de conserver le pouvoir dans les mains d'une droite " forte ", mais aussi de faire face à la force grandissante des ouvriers, en particulier ceux qui appartenaient aux usines de pointe comme celles du secteur métallurgique et chimique.

L'identité d'une nation dépend, on le sait, du dialogue qui se tisse et se maintient entre différentes voix. Or, la dictature qui a précédé la démocratie au Brésil n'offrait qu'un discours unique, autoritaire et monophonique. Pour que le dialogue puisse être à nouveau instauré, il fallait une rupture: l'ébauche et puis la création effective du nouveau parti de Lula est sans aucun doute le fruit d'une rupture historique. La formation de ce parti peut être décrite comme suit :

Le PT surgit sans une idéologie précise, mais il a le mérite de contenir en son sein des chefs religieux progressistes, des intellectuels et des sections qui s'opposaient à la dictature, des organisations marxistes-léninistes et les dirigeants du «nouveau syndicalisme » [...] ( Garcia $2000: 18)^{3}$.

11 Quoi qu'il en soit, le PT apparaît - et c'est cela qui le différencie des autres partis - au gré d'un mouvement « nouveau » en politique brésilienne, puisqu'il est n'est pas né du bon gré de l'élite ou de la bourgeoisie : il surgit grâce à un mouvement "contraire ", venu des classes considérées dans l'échelle sociale comme "inférieures ${ }^{4}$.

Revenons à Lula. Si un mouvement politique comme le RPF (Rassemblement du Peuple français) organisé en France par De Gaulle, le 14 Avril 1947, doit énormément aux actions du Général pendant la seconde Guerre Mondiale, on peut dire que dans le cas de Lula, mutatis mutandis, la même chose s'est passée. Lula jouissait en effet de la légitimité que lui conférait son poste de dirigeant syndicaliste ${ }^{5}$, qui lui garantissait une influence grandissante auprès de ses collègues. Et aux paroles, il a ajouté des actions positives qui ont fait contraste avec les actions négatives soutenues par le régime militaire d'extrême droite qui a assombri le pays pendant près de 30 ans. 

de grève ouvrière, ce que lui a valu un séjour de 31 jours en prison: le leader syndicaliste avait enfin conquis la trempe du héros. On ne peut oublier non plus que Lula a également participé à la fondation de la CUT - Centrale unique des Travailleurs, en août 1983. Et qu'il est enfin entré dans la vie politique, à proprement parler, en se faisant élire député fédéral en 1986. Quelle victoire pour un ouvrier ${ }^{6}$ ! Il s'est porté candidat à la Présidence du Brésil pour la première fois en 1989, juste après le retour de la démocratie au pays, après vingt-neuf années, durant lesquelles le peuple n'a pas eu droit au suffrage universel.

En 1994 et en 1998 Lula s'est à nouveau porté candidat mais il a été battu par Fernando Henrique Cardoso. Finalement, le 27 octobre 2002, à 57 ans, avec presque 53 millions de voix, Lula a été élu président du Brésil ; quatre ans après, il a été reconduit dans le même poste.

\section{Récits de vie et discours politique : un agencement gagnant?}

\subsection{Réflexions sur un genre discursif : le récit de vie}

Comme on l'a déjà noté, le récit de vie est un genre qui circule déjà depuis un certain temps dans le champ des sciences sociales. On peut ainsi le voir entre 1918 et 1920, les années où deux sociologues (de l'Ecole de Chicago) William Thomas et Florian Znaniecki ont fait paraître un ouvrage (en 5 tomes) intitulé Le paysan polonais en Europe et aux États-Unis (The Polish Peasant in Europe and America), qui mettait l'accent sur les « récits de vie ", et s'intéressait à l'organisation sociale et à l'évolution d'un groupe dans une société étrangère. Ce type «narratif » perçu comme une méthodologie de recherche a été introduit en France par Daniel Bertaux, et selon les différents courants ou disciplines qui s'en sont emparés, il a reçu des dénominations variées : histoire de vie, récit de vie, narration de soi ou autobiographie (Orofiamma $2008: 68-81$ ). Quelle que soit la variété des expressions utilisées, il faut que l'acte de se raconter soit volontaire pour que le récit ne ressemble pas à un interrogatoire policier. Toujours est-il que, « en se racontant, il [le sujet-énonciateur ou narrateur] met aussi en scène une dramaturgie personnelle qui le présente en tant que héros glorieux d'une histoire dont il a surmonté les obstacles » (Orofiamma 2008: 80-81). Parler de sa vie passée en public constitue donc une tâche courageuse : le récit de vie construit la petite " épopée » d'un être issu d'un quotidien banal. Lipiansky (1983:55) nous en donne l'intéressante définition que voici :

Le récit de vie est une tentative du sujet pour construire et donner une image de lui-même. [...] C'est l'effort pour ressaisir son identité à travers les aléas et les avatars de l'existence dans une cohérence qui la rend communicable à autrui. Le récit suppose ainsi un processus de totalisation, à travers lequel l'énonciateur cherche à donner sens et consistance à sa vie.

En ce sens, l'acte narratif ne va pas sans rappeler l'acte de se raconter en psychanalyse : au fur et à mesure que l'on parle de soi, on se libère et l'on peut mieux s'accepter ou accepter sa "vérité », engloutie dans un passé plus ou moins refoulé que l'on s'efforce de reconstruire. D'ailleurs, comme l'affirme Abastado, « il s'agit moins de retrouver le passé que de faire exister ce qu'on affirme pour se donner une identité. Narro, ergo sum 
[...]. Les jeux de la mémoire et de l'oubli, le maquillage des souvenirs dessinent une image du locuteur conforme à son idéal » (1983:7).

Parmi les théoriciens du domaine de la linguistique qui ont utilisé le récit de vie dans leurs travaux, avant que ce genre narratif ne revienne avec force, aujourd'hui, à travers les études de biographies ou d'autobiographies, il faut citer les travaux d'Henri Boyer (1988) qui emprunte certains concepts à Abastado (1983) et analyse le genre à la lumière des travaux de Weinrich (1973). Boyer étudie des récits de vie qui ont été publiés dans trois hebdomadaires français différents, et qui font partie du «Courrier des lecteurs " de chaque journal. Ce sont donc des textes qui racontent la vie d'êtres ordinaires, sortis momentanément de leur anonymat pour venir se «confesser » dans cette rubrique. Il affirme :

L'intention de communiquer un vécu, mais aussi la volonté de revendiquer une identité [...] passent par tout une mise en scène, par un incontournable travail d' écriture, par un effort plus ou moins important pour produire un texte conforme à une certaine représentation de la narration écrite (Boyer 1988 : 55-56).

Nous sommes loin du récit de vie spontané, inséré dans l'oral. Ici la question est plutôt centrée sur l'effort de gens simples qui s'appliquent à bien écrire puisqu'ils s'adressent à leur journal et se sentent ainsi plus ou moins obligés de faire un travail qui pourrait être proche d'une "pratique scolaire" (Abastado 1983: 11-12). En effet, Boyer va se pencher sur la question de l'utilisation des temps verbaux, en prenant comme point de départ le modèle d'opposition entre commentaire et récit dû à Weinrich (1973). Ce faisant, il réalise une analyse basée sur la grammaire textuelle. Mais sa démarche est intéressante de notre point de vue car elle a le mérite de présenter des spécificités langagières du genre et de montrer comment l'alternance des temps au passé simple/ passé composé peut avoir une finalité subjective de la part de celui qui signe son « récit de vie».

19 «L'intention de communiquer un vécu, mais aussi la volonté de revendiquer une identité » : retenons ces éléments, qui font actuellement partie des préoccupations de l'AD lorsqu'elle traite du genre en question. Qui plus est, cette affirmation ouvre sur le projet de parole du sujet-communiquant ou narrateur du récit de vie. L'intention de communiquer n'échappe pas à une certaine «littérature» comme forme de construction d'un récit "élaboré » (Abastado 1983 : 11-12). Dans cette perspective, on peut dire que dans tout récit de vie il y une construction discursive. Sans doute est-elle plus élaborée s'il est écrit, plus spontané s'il est oral ; mais ce qui importe vraiment, c'est que le passé est reconstruit et réélaboré dans la mise en mots. En d'autres termes, toute reconstruction du passé d'un individu oscille entre la mise en place d'effets de réalité et d'effets de fiction, stratégie qui fait alterner, selon Charaudeau, « deux modes de vision du monde » (1992: 695), et qui - tout en aidant le locuteur à construire une image de soi - fait tout naturellement partie du souvenir.

Si l'on revient à l'objet de cet article - le « pourquoi » des récits de vie de Lula insérés dans des segments de ses discours politiques -, on peut dire qu'en se racontant, il a voulu faire accepter son identité d'ex-ouvrier et gamin pauvre, ou l'imposer à l'« autre ", mais aussi à lui-même. On insistera ici sur le travail d'élaboration de la mémoire, qui recrée les événements en les reconvertissant en mots. Si on parle du passé, comme Lula l'a fait, on puise dans des souvenirs ; ils ont beau être vrais et avérés par d'autres, le travail de reconstitution du passé donne au récit des couleurs proches des récits de fiction grâce à l'inscription de la subjectivité dans le discours, au point de 
vue emprunté par le narrateur. Tout récit de vie comporte donc ces doubles effets de réalité et de fiction, car ils font partie de l'action de se raconter. Il y va, au fond, d'une curieuse stratégie propre aux êtres humains et qui procède dans deux sens : celui du sujet-communiquant et celui du sujet-interprétant ${ }^{7}$. Dans le cas spécifique de Lula : par l'évocation d'un passé de luttes et de pauvreté qui a été couronné par une ascension glorieuse vers la présidence, Lula a cherché à émouvoir son public tout en s'émouvant lui-même.

\subsection{Les renvois indirects au récit de vie dans les allocutions politiques de Lula}

21 Si on jette cependant un regard rapide sur quelques extraits du premier discours d'investiture de Lula, en $2003^{\circ}$, on dira qu'il ne lie pas (à une exception près) sa victoire à sa trajectoire de vie. Le discours présidentiel du premier mandat semble avoir une dimension plus large, il s'ouvre au peuple sans se replier sur le passé d'un individu. En ce sens, on peut citer, en guise d'exemple, un énoncé « neutre » parmi tant d'autres :

(2) [...] Il faut accroître - et de beaucoup - le marché intérieur, le renforcement des petites et des micros entreprises. Il faut également investir dans la formation et l'infrastructure technologique consacrée à l'écoulement de la production. ${ }^{9}$

Il faut expliquer ce que l'on comprend par " neutre" dans cette situation de communication. Au cours d'une lecture cursive, on pourrait - un peu hâtivement considérer l'énoncé ci-dessus comme issu des discours qui relèvent de la «langue de bois » adoptés par de nombreux hommes politiques de par le monde. Il est en effet courant, dans le discours politique, de promettre des améliorations, surtout lors des occasions solennelles. Et comme on peut le voir, le contenu de ce segment ne fait pas partie du récit personnel de la vie de Lula gamin ou adolescent pauvre. On peut cependant supposer qu'il appartient aux rêves du jeune Lula syndicaliste et fondateur du PT. Un autre fait curieux lié à ce segment, c'est que les actes de langage qui y apparaissent ont, avec le temps, assumé le rôle d'actes performatifs : en effet, le gouvernement Lula les a effectivement accomplis. En d'autres termes, le pays a progressé avec Lula et, curieusement, dans le sens de ce qui a été dit dans le segment (2).

23 Le premier discours d'investiture de Lula, en 2003, est moins lié que celui du second mandat à son récit de vie de gamin pauvre : récemment élu, Lula a pris des précautions pour donner à ses paroles un ton plus solennel. Mais le renvoi indirect à des éléments de son récit de vie n'est pas passé inaperçu aux yeux de ceux qui suivaient déjà sa vie politique et connaissaient sa tendance à parler avec effusion devant différents auditoires. Dès lors, même dans ce premier discours d'investiture, on a pu apercevoir des bribes qui renvoient à son histoire de vie personnelle.

Mon hypothèse est que certains sujets communicants laissent plus que d'autres, de façon volontaire (ou non), des marques énonciatives plus ou moins visibles qui renvoient à leur "vraie» vie en tant que sujets historiques, et que ces traces constituent des messages adressés aux auditeurs ou lecteurs ou encore, le cas échéant, aux sujets-parlants eux-mêmes, dans un procédé dialogique interne. «Si je m'entends dire cela je me sentirai plus proche de moi-même (de mon "moi" profond) et j'aurai plus de chance de m'accepter et d'être accepté ", semblent-ils dire. En ce sens, le fait de se raconter peut libérer le sujet-racontant de certaines angoisses et le préparer à une 
meilleure acceptation de son passé ; cette affirmation, que la psychanalyse pourrait reprendre à son compte, semble aussi convenir à certains cas liés au genre du récit de vie.

L'énoncé suivant, qui appartient également au même discours d'investiture de Lula, est en ce sens ambigu : est-il ouvert à toute une société ou fait-il référence à des aspects du «moi-Lula»?

(3) «Changement »; tel est le mot clé, cela a été le grand message de la société brésilienne dans les élections d'octobre. L'espoir l'a, finalement, emporté sur la peur, et la société brésilienne a décidé qu'il était temps d'emprunter de nouvelles voies $^{10}$.

Plusieurs mots de la classe des «axiologiques" peuvent être relevés dans cet énoncé. Commençons par le substantif «mudança/changement»; le «changement» en question parait, au premier abord, lié à une transformation politique collective; en outre, ce segment, placé juste dans les toutes premières lignes du discours, semble englober la « voix » du PT, en l'occurrence du parti, qui s'immisce dans celle de Lula un parti qui va pour la première fois (depuis sa création) diriger le pays. Le syntagme "nouvelles voies» peut être le symbole de cette voix dédoublée. Mais le "changement » dont il est question peut également se rapporter à une modification individuelle qui affecte un individu prédestiné : Lula. On doit convenir qu'il a changé son destin en osant créer le PT : et une fois cet acte consommé, le PT a doté Lula de certains devoirs : par exemple, celui de se présenter aux élections présidentielles de son pays. Cela a fait rire certains Brésiliens, lors des premières tentatives de ce " petit » candidat, représentant d'un " petit » parti : très peu d'entre eux acceptaient alors ce " changement de cap » et auraient oser confier la présidence du pays à un syndicaliste qui était même appelé, très méchamment " anarfa », un diminutif très péjoratif usité en portugais pour désigner un analphabète ou quelqu'un qui ne parvient pas à prononcer correctement les mots ${ }^{11}$. Mais, le temps aidant, les Brésiliens ont changé d'opinion aussi bien sur le Parti des Travailleurs que sur son dirigeant. Ainsi, le « changement » énoncé par Lula acquiert un sens métonymique : tout un passé personnel de luttes, d'échecs et finalement de succès y est contenu.

Toutefois, si on s'arrête sur cette ambivalence ou ce «flou discursif », on peut déceler dans le discours du Président un caractère populiste : il y a là comme « un appel au peuple, appel à engendrer un élan collectif, à se dépasser pour en finir avec une situation de décadence, appel à suivre le sauveur qui le fera renaitre dans un monde meilleur" (Charaudeau 2008: 83). La référence à des sentiments très forts comme " espoir/esperança » et "peur/medo ", liés à la trajectoire de vie de l'ex-ouvrier, n'est pas aléatoire : le discours assume une dimension argumentative.

Mais la peur, rappelons-le, peut engendrer l'action. Pour Braud « ce qui distingue les différents acteurs politiques, c'est le choix des peurs et, surtout, l'intensité de l'activation émotionnelle » (2007 : 266). En racontant son histoire et en mentionnant sa peur qu'il joint habilement à l'espoir, Lula fait aisément circuler son discours entre l'individuel et le collectif. L'individuel: lui, le Brésilien pauvre, sans diplômes universitaires qui est arrivé à la présidence. Le collectif: des millions de Brésiliens démunis qui l'ont vu comme un des leurs et qui lui ont permis d'être élu, qui l'ont appuyé, en associant l'image de Lula (celle de sa vie avant les victoires politiques) à la leur. Ainsi, dans le segment (3), la voix présidentielle se démultiplie et présente plusieurs facettes. Cela est également perceptible dans le jeu entre le «je/moi » du 
Président et le "nous » qui se veut «fédérateur", dans cet autre extrait du même discours :

(4) Le Brésil est un pays immense, un continent d'une grande complexité humaine, écologique et sociale, avec presque 175 millions d'habitants. Nous ne pouvons pas le laisser aller à la dérive, au gré des vents, sans un véritable projet de développement national [...]. Si nous voulons le transformer, vivre dans un pays où tout le monde peut marcher la tête haute, nous devons exercer tous les jours deux vertus : la patience et la persévérance ${ }^{12}$.

Dans (3) et (4) on voit que Lula ne se sert pas de la première personne du singulier et se positionne derrière un « nous » fédérateur égal à : moi Président, vous qui m'avez élu et notre pays, le Brésil. Ce faisant, il opère une relecture et une reconstruction de l'Histoire du Brésil et se montre comme un personnage (parmi d'autres) de celle-ci. Le «nous » et le « on » du sujet parlant se rejoignent dans une seule entité qui se propose de travailler pour la collectivité. Mais c'est seulement à première vue : en effet, la présence de deux sentiments très allégoriques comme "patience » et "persévérance » conduisent la lecture vers un autre possible interprétatif. Lula fait appel à des sentiments "bon marché ", à des "lieux communs ». Y-a-t-il, ici, référence au trajet personnel de Lula? Et n'est-il pas en train de parler en répondant à l'attente de ceux qui voulaient entendre justement ce type de discours ${ }^{13}$ Sans doute. La preuve en est que c'est avec de semblables discours qu'il a réussi à toucher son grand public d'électeurs.

\subsection{Usages explicites du récit de vie dans le discours politique de Lula}

Il répond donc à l'attente de son auditoire - et « s'auto-légitime » comme celui qui sera capable de promouvoir le changement requis par ses électeurs. Ainsi,

(5) Chacun de nous, Brésiliens, vous savez que ce que nous avons fait aujourd'hui n'est pas peu, mais vous savez que l'on peut faire mieux. Quand je pense à ma propre vie de migrant du Nord-Est du pays, garçon qui vendait des arachides et des oranges sur les quais du port de Santos, qui est devenu un mécanicien tourneur et un dirigeant syndical, qui a fondé le Parti des Travailleurs, qui a eu confiance en ses actions et qui assume aujourd'hui le poste de Haut représentant de la nation, [devant tout cela] je sais avec clarté et avec pleine conviction, que nous pouvons faire plus. ${ }^{14}$

31 Deux sujets ou deux rôles sont ici assumés par le sujet parlant. Dans les deux premières lignes il se masque en sujet énonciateur qui se confond avec le peuple à qui il s'adresse, par le «nous/on». Le «je» ne fait son apparition qu'à la troisième ligne pour annoncer le « récit de vie» du sujet. Il y effectue alors une sorte de digression pour raconter une partie de son "chemin de croix». Et il se sert d'une synecdoque pour la raconter : il "construit» la "petite histoire» dont l'acteur principal est l'ouvrier devenu Président. Or, la «petite histoire » fait écho à celle de milliers de Brésiliens comme lui, des êtres miséreux, affamés, fuyant la sécheresse d'une région pauvre du Brésil, migrés comme lui dans cette sorte d'Eldorado, l'état le plus riche et le plus industrialisé du Brésil : São Paulo.

On note encore, dans le récit de vie, la présence d'un déplacement temporel et situationnel. Tout d'abord, enfant pauvre de Pernambouc, ensuite petit vendeur 
d'arachides et d'oranges sur le quai du port de Santos, puis syndicaliste à São Bernardo do Campo, et enfin Président de la République.

Ce que l'on tient à affirmer, c'est que le récit de vie a une fonction ou une «dimension argumentative ». Comme le dit Amossy : « il y a aussi des discours qui ne se donnent pas comme des entreprises de persuasion déclarée, [...]. L'argumentation n'y est ni apparente, ni explicite » $(2006: 245)$; mais celle-ci existe pourtant : elle apparaît dans l'orientation conférée au discours par son auteur, lorsqu'il essaie de "projeter un certain éclairage sur ce dont il traite» (ibid.). Cette dimension argumentative, bien propre au récit de fiction avec son caractère atemporel et magique - dans le cas de Lula: le crapaud qui devient Prince -, vient ici donner plus de force à la "visée argumentative » qui commande l'ensemble d'un discours politique. Ainsi (5) pourrait être considéré à travers un schéma assez simple : Si A, donc B, soit : «Si j'ai vaincu la disette, donc vous êtes capables d'en faire autant ». Voilà donc le noyau ou le thème de ce récit de vie : la fusion entre l'histoire de Lula avant son élection, sa " petite histoire " (qui s'accorde avec l'état défavorisé de millions de Brésiliens) insérée dans la «grande Histoire » du Brésil ou le passage réussi qu'il a entrepris d'une «culture de pauvreté » vers une « culture de transformation».

En effet, pour mieux comprendre Lula et ses discours, il faut se pencher sur ces deux syntagmes, dus à l'anthropologie et largement repris et expliqués par Paraná (2008: 367-454). En synthétisant, on peut dire que la culture de pauvreté représente un mode objectif et précis de survie adopté par des groupes marginalisés ou semi-marginalisés dans une société qui valorise l'argent et le pouvoir. Cette culture imprègne totalement les individus : elle leur colle à la peau et se laisse saisir dans leurs gestes et paroles comme dans la sensation d'être exclus et méprisés par les institutions de l'Etat. Elle est aussi responsable d'un sentiment de peur éprouvé par les pauvres et les démunis face à un monde hostile. Comment avoir une conscience de classe dans des telles conditions et ne pas se laisser porter par le conformisme? Lula, qui a fait partie d'un groupe défavorisé et donc de cette culture de pauvreté dans son enfance et son adolescence, a peu à peu acquis une conscience de classe à travers son insertion progressive dans d'autres groupes culturels : tout d'abord, il a dû apprendre à vivre dans une grande métropole, ensuite il a dû apprendre à communiquer avec ses collègues ouvriers et, finalement, il a dû assumer les tâches d'un leader syndical. Un homme qui occupe ces fonctions doit savoir parler de façon convaincante. Il a ainsi préparé le terrain pour se libérer de la culture de pauvreté. A chaque nouveau pas, son discours et sa façon de communiquer avec le monde se sont transformés ou adaptés aux nouvelles circonstances de sa vie. Or, "la culture de transformation rassemble ce qu'il y a de mieux dans les énergies créatrices", dit Paraná (2008: 449); elle contribue à faire apparaître « des désirs et des actions plus solidaires et collectifs » (ibid.). Cela explique, croit-on, le désir de créer un parti politique et de le mettre au service de tous les Brésiliens et pas seulement d'une élite.

L'assurance d'être déjà entré dans une autre étape de vie est visible dans le second discours d'investiture, prononcé le $1^{\text {er }}$ janvier 2007 ; Lula peut ici exhiber son ethos de «Président du peuple » et raconter son histoire, non sans la fierté de ceux qui ont su vaincre la culture de pauvreté pour entrer dans la culture de transformation :

(6) Ici même, il y a quatre ans, un premier Janvier, j'ai connu la plus importante expérience de ma vie - celle d'assumer la présidence de mon pays. Il ne s'agissait pas seulement de la réalisation d'un rêve personnel. Ce qui s'est produit est le résultat d'un puissant mouvement historique, dont je ne me sentais qu'un humble 
instrument, et j'ai toujours ce sentiment. Pour la première fois, un homme qui est né dans la pauvreté, qui a dû lutter contre le risque de la mort pendant son enfance et de vaincre son désespoir quand il est devenu adulte, [cet homme] est arrivé au plus haut poste de la République, à travers une élection démocratique. Pour la première fois, le long chemin de croix d'un migrant très pauvre qui avait commencé son chemin comme celui de millions de gens venus du Nord du Brésil, en haut d'un camion qui transportait les voyageurs debout, aboutissait à l'expression d'un projet collectif, dans la montée vers le Palais de la Présidence de la République ${ }^{15}$. ré-investiture du Président, un certain ton messianique qui peut frôler le " populisme » (Charaudeau 2008). Certes, Lula est sincère - il ne ment pas sur son enfance pauvre mais il clame un peu trop haut et fort son entrée dans la " culture de transformation ». Quel est son but? Exorciser son passé de malheur? Et, ce faisant, cherche-t-il à émouvoir son auditoire et à se faire accepter par tous? Sans doute. Cependant, sous le «je» de Lula ou dans ces multiples «je» on peut voir en filigrane la présence d'un « on » collectif et social qui le lie véritablement à une communauté. C'est en cela que son discours diffère d'une parole purement " populiste ».

Ce second discours d'investiture se clôt sur une "prière ». Mais est-ce bien une prière ? Ou plutôt une invocation de la divinité ? C'est Lula lui-même qui a demandé son inclusion. On le sait croyant. On sait que le Brésil est un pays chrétien. On sait qu'en terres brésiliennes les gens demandent la protection et la bénédiction de Dieu à chaque instant de leur vie, n'en déplaise aux intellectuels du pays qui gardent généralement leurs distances par rapport aux croyances religieuses. Voici la «prière » en question :

(7) Mesdames, Messieurs, /Je reconnais que Dieu a été généreux avec moi. /Plus que je ne le mérite. /J'ai demandé de la force... et Dieu m'a donné des difficultés à surmonter pour que je devienne fort /J'ai demandé de la sagesse... et Dieu m'a donné des problèmes à résoudre. /J'ai demandé de la prospérité... et Dieu m'a donné un cerveau et des muscles pour travailler. /J'ai demandé du courage... et Dieu m'a donné des dangers à affronter. /J'ai demandé de l'amour... et Dieu m'a donné des gens que je devais aider. /J'ai demandé de la chance... et Dieu m'a donné des opportunités pour la saisir. /Je n'ai rien reçu de ce que j'avais demandé mais j'ai reçu tout ce dont j'avais besoin ${ }^{16}$.

La prière a le mérite (en ce qui concerne la captation d'un auditoire composite) d'être assez œcuménique, et elle clôt le discours. Elle se base sur des proverbes comme « $\mathrm{Ne}$ nous plaignons pas du mal, il vient de Dieu», et d'autres adages populaires qui réunissent les deux faces d'une situation, d'où une certaine oscillation entre le "positif » et le «non-positif». Somme toute, Dieu est le grand ironiste qui a toujours raison. Bref, dans la «prière » du discours de Lula, on voit l'homme qui se soumet aux caprices de la divinité et reconnaît qu'ils ont eu une raison d'être. Comme l'ange voltairien apparu au personnage de Zadig, l'homme doit se plier aux aléas du destin. Ainsi, le discours de Lula qui appartient, dans son ensemble, au genre "politique" introduit dans celui-ci un nouvel "effet de genre» (Charaudeau 1992: 698) qui serait plutôt approprié aux allocutions prononcées lors des rencontres «religieuses». On insiste sur le fait que cette prière a été choisie par Lula : cela montre l'expression d'une modalité énonciative assumée par un sujet parlant précis. En d'autres mots : ici, on a perçu l'expression d'une attitude du locuteur Lula et cette attitude fait partie de ses convictions intimes, celles d'un homme qui croit vraiment en Dieu; il semble qu'il veut que cela transparaisse dans son discours (Machado, 2001: 64). Ainsi, en suivant la terminologie chère à Amossy, on peut dire que l'énoncé (7) revêt une «dimension 
argumentative »(2006). Le frère, l'égal des ses concitoyens : Lula assume encore une fois un «ethos d'humanité » (Charaudeau 2005). Et l'humanité ou la générosité chez Lula semblent lui avoir été léguées par sa mère, figure émouvante de sa vie.

\section{Une mère et son fils}

La mère de Lula a une place assurée dans ses discours, de façon générale. On en a déjà vérifié une occurrence ici-même. En voici deux autres, extraites d'un discours de campagne prononcé en 2002, en pleine Avenue Paulista (une importante artère de la ville de São Paulo) :

(8) Je me rappelle de ma mère qui avait peur que j'entre dans le Syndicat, peur que je sois mis en prison. Elle est morte et j'étais en prison, et elle ne savait pas que j'avais été arrêté. Ces gens qui sont morts et qui ne peuvent pas être avec nous, maintenant, vous pouvez être sûrs qu'ils sont là-haut en train de nous observer, de rire de joie, parce que nous avons réussi à construire le rêve de quelques générations ${ }^{17}$.

(9) Et je veux que vous sachiez que ma mère disait [silence] ma mère est née et décédée analphabète, et ma mère a dit " mon fils, la seule chose que l'homme ne peut pas perdre est le droit de marcher la tête haute et de regarder dans les yeux les gens avec qui il parle $[. ..] »^{18}$.

Le ton utilisé dans le discours de 2002 et dans les extraits ci-dessus vient du discours oral, improvisé, d'où quelques façons de s'exprimer propres à la conversation : pauses, répétitions, silences, énoncés non conclus, reprise de la parole et propos mélangés. Voyons (8) de plus près : Lula commence par une évocation de la peur de la mère, lie ce souvenir à celui de son emprisonnement et finit par évoquer des morts (dont sa mère) qui sont « là-haut », c'est-à-dire, dans le paradis chrétien (le ciel), donc absents de ce monde mais convoqués par les paroles de l'homme politique à regarder la gloire d'un fils ou frère brésilien qui sort enfin de la culture de pauvreté ou de la marginalité politique. Les morts évoqués par Lula sont, on s'en doute, tous ceux que la dictature du pays a fait sommairement disparaître et parmi eux, des camarades ouvriers. Il faut convenir que cet amas de souvenirs acquiert une cohérence à la fin de (8) : Lula réussit une fois de plus par la stratégie qui consiste à mettre en scène des pans de son récit de vie, à confirmer son ethos de Brésilien populaire.

Dans (8), on peut observer une curieuse construction langagière et qui pourrait elle aussi, dans d'autres circonstances et dans la voix d'autres hommes politiques, passer pour un simple appel au pathos de l'auditoire: «Elle [ma mère] est morte et j'étais en prison ». Il s'agit néanmoins d'une histoire vraie. Et d'après son récit de vie (Paraná 2008), on sait qu'il était l'enfant chéri de cette mère qui l'a toujours épaulé et qui savait que son fils changeait de chemin mais, en même temps, qu'il irait affronter de nouveaux périls. En (9) on voit que Lula, comme tant des Brésiliens issus de la culture de pauvreté, n'a pas honte de dire que sa mère était illettrée mais sage. Le conseil qu'elle lui a donné ( "mon fils, la seule chose que l'homme ne peut pas perdre est le droit de marcher la tête haute et de regarder dans les yeux les gens avec qui il parle ») est sans doute issu d'un énoncé devenu lieu commun chez les humbles du Brésil. Elle a beau être stéréotypée, elle a un impact argumentatif considérable car ce stéréotype a des racines dans la nuit des temps, des temps brésiliens, il faut le préciser : l'esclavage a sévi dans le pays jusqu'en 1878 et les esclaves (Noirs, Indiens, Métis) n'avaient pas le droit de regarder le seigneur blanc dans les yeux. Aujourd'hui, on peut être pauvre et 
affamé, on n'est plus esclave et on doit affronter la vie la tête haute et ne plus baisser le regard si on est honnête et travailleur.

Toutefois, la mention répétée de la figure maternelle peut sembler pour le moins curieuse si l'on songe à la figure du père, le grand absent de cette grande confession publique. Comment expliquer ce phénomène ? La réponse à cette question ressort de la lecture des récits de vie de Lula et de sa famille que Paraná (2008) a recueillis et analysés dans l'optique de l'histoire orale. C'est à travers la voix de Lula que l'on apprend que son père "tyrannique mais travailleur » (ibid. : 50) était très dur avec Lula, ses frères et sa mère ; qu'il était, en somme, un grossier personnage. Or, un jour, dans une crise de furie, ce père a voulu sans raison aucune brutaliser son fils cadet, Lula. La mère - jusque-là apparemment résignée sur son sort (vivre avec un mari qui avait une seconde famille, subir sa violence, etc.) - s'est enfin insurgée contre lui et n'a pas permis qu'il malmène le petit. Et ainsi, elle a eu le courage de quitter son mari et d'aller vivre seule avec ses enfants. Cela peut expliquer, au moins en partie, le pourquoi de l'enthousiasme de Lula à l'égard de sa mère et des mères en général, au détriment de la figure paternelle.

Là encore, cependant, on voit la formation d'une stratégie persuasive. La trajectoire de Lula et de sa mère emboîte le pas à celle d'autres mères brésiliennes ${ }^{19}$ et confirme la fusion de la petite histoire personnelle du sujet-communiquant avec la grande Histoire de son pays.

\section{Quelques mots en guise de conclusion}

Cette analyse met en évidence la voix d'un personnage qui veut communiquer efficacement par le moyen des discours politiques en mobilisant éventuellement des bribes de son histoire personnelle et en revendiquant ainsi son identité.

Le récit de vie en tant que genre n'a pas une visée argumentative précise: mais il contient une dimension argumentative, que l'on trouve parfois dans la littérature ou dans les récits qui mélangent des effets de fiction aux événements réels (chroniques, feuilletons, faits divers, parmi d'autres). L'argumentation du récit de vie désigne alors plutôt l'orientation (qui n'est pas toujours déclarée ou visible) que le sujetcommuniquant ou narrateur veut imprimer à son discours (Amossy $2006: 245$ ). Ainsi, dans le cas de Lula, le récit de vie avec sa dimension argumentative a pénétré dans son discours politique, doté d'une visée argumentative précise. Cela a doté l'ensemble discursif d'une tonalité particulière. En plus, se raconter en public - tâche qui n'est ni aisée, ni aléatoire - ne se fait pas sans le concours de l'émotion. Naturellement inscrite dans le récit de vie de Lula, celle-ci n'a pas eu de peine à traverser ses discours politiques et à susciter un effet de pathos auprès de son auditoire.

Reste la question de savoir si cela pointe ou non vers une forme de "publicité populiste " d'un "héros du peuple» au sens que Charaudeau (2008) donne à ces expressions. Nombreux sont ceux qui pensent au Brésil que Lula n'a pas eu besoin d'un discours ou d'une publicité "populiste" pour arriver au pouvoir et recevoir $84 \%$ de l'approbation de l'opinion publique de son pays, toutes classes sociales confondues. La simplicité de ses dits n'apparaît pas dans ce cas spécifique comme une simple stratégie de séduction : elle entre en unisson avec les actions de Lula depuis qu'il a commencé sa carrière politique jusqu'à la fin de son mandat comme Président de la République, et contribue à projeter un ethos crédible. En même temps, il faut bien voir que la scène 
discursive où résonne la voix de Lula contient (comme toutes les scènes discursives politiques) la présence de maintes stratégies de captation. En faisant appel à son histoire de vie, Lula a sans aucun doute senti, en orateur attentif, qu'il possédait là un excellent moyen de captation de son auditoire. En effet, on l'a vu, son histoire personnelle contient des éléments qui justifient la présence de l'émotion dans ses discours. Qui plus est, elle se confond - dans ses moments les plus durs - avec celle des déshérités du Brésil, renvoyant aussi aux troubles qui ont secoué le pays à l'époque la plus obscure son histoire politique, celle des « années de plomb » de la dictature.

\section{BIBLIOGRAPHIE}

Abastado, Claude. 1983. « Raconte ! Raconte... les récits de vie comme objet sémiotique ", Revue des Sciences Humaines 191, 5-21

Amossy, Ruth. 2006. L'argumentation dans le discours (Paris : Colin)

Bertaux, Daniel. 1976. Histoires de vie - ou récits de pratiques? Méthodologie de l'approche biographique en sociologie (Rapport au CORDES)

Bertaux, Daniel. 1980. «L'approche biographique, sa validité méthodologique, ses potentialités », Cahiers internationaux de sociologie 69, 198-225

Bertaux, Daniel. 2006. Le récit de vie (Paris : Colin)

Boyer, Henri. 1988. L'écrit comme enjeu. Principe de scription et principe d'écriture dans la communication sociale (Paris : Didier)

Braud, Philippe. 2007. Petit traité des émotions, sentiments et passions politiques (Paris : Colin)

Bruner, Jérome. 2002. Pourquoi nous racontons-nous des histoires? Le récit au fondement de la culture et de l'identité individuelle (Paris : Pocket)

Charaudeau, Patrick. 2008. Entre populisme et peopolisme. Comment Sarkozy a gagné (Paris : Vuibert)

Charaudeau, Patrick. 2005. Le Discours politique. Les masques du pouvoir (Paris : Vuibert)

Charaudeau, Patrick. 1992. Grammaire du sens et de l'expression (Paris : Hachette)

Charaudeau, Patrick. 1983. Langage et Discours (Paris : Hachette)

Garcia, Cyro. 2000. Partido dos Trabalhadores : rompendo com a lógica da diferença (Niterói : Dissertação deMestrado, Universidade Federal Fluminense)

Lipiansky, Marc. 1983. « Une quête de l'identité », Revue des sciences humaines 191, 61-69

Lula Da Silva, Luiz Inácio. 2003, 2007. Discursos de posse - $1^{\circ}$ e $2^{\circ}$ mandatos. [En linge : http:// www1.folha.uol.com.br/folha/brasil/ult96u44275.shtml, et http://www.fenecon.org.br/ DiscursoLula2007.pdf; consultés le 25 juillet 2011]

Lula Da Silva, Luiz Inacio, 2002. Discurso realizado na Avenida Paulista em28 de outubro de 2002. [En ligne : http://noticias.terra.com.br/eleicoes/interna/0,OI64590-EI380,00.html; consulté le 25 juillet 2011] 
Machado, Ida Lucia. 2001. «Breves considerações sobre índices de modalização \& práticas de leitura », Caligrama 6, 63-77

Orofiamma, Roselyne. 2008. « Les figures du sujet dans le récit de vie - en sociologie et en formation », Informations sociales 145, 68-81

Paraná, Denise. 2008. Lula, o filho do Brasil (São Paulo : Editora Fundação Perseu Abramo, 3ª edição) Salmon, Christian. 2007. Storytelling, la machine à fabriquer des histoires à formater les esprits (Paris : La Découverte)

Weinrich, Harald. 1973. Le temps (Paris : Seuil)

\section{NOTES}

1. C'est-à-dire, deux mandats consécutifs.

2. Voir Paraná, Denise. 2008. Lula, o filho do Brasil. Dans ce livre, l'auteure a rassemblé de nombreux entretiens qu'elle a réalisés avec Lula et quelques membres de sa famille, dans lesquels ceux-ci racontent leurs vies dans le Nord du Brésil et puis à São Paulo et surtout, dans le cas de Lula et de son frère surnommé «Frei Chico » (celui à qui Lula doit en grande partie comme luimême l'affirme dans le livre, son "initiation" dans la vie politique), leur trajectoire dans la politique brésilienne. Le ton du livre se situe entre biographie et l'histoire orale : l'auteure tient à montrer comment le récit de vie d'une famille pauvre (les «Silva») s'imbrique et explique une partie importante de l'histoire du Brésil.

3. En portugais : «O PT surge sem uma ideologia precisa, mas tem o mérito de conter no seu interior as lideranças da Igreja progressista, intelectuais e setores da classe média que se opunham à ditadura, organizações marxistas-leninistas e as lideranças do "novo sindicalismo" [...]».

On remarque chez Garcia une observation bien juste : «Le PT surgit sans une idéologie précise ». En effet Lula et bien d'autres qui ont adhéré au mouvement petista n'ont pas suivi les voies naturelles qui normalement façonnent la personnalité des politiciens de gauche. Cela est sans doute contradictoire dans le cas de Lula qui est pourtant vu par certains comme un homme politique appartenant à une faction de la « gauche radicale » du Brésil ! Ce qui n'est pas du tout vrai : Lula l'affirme d'ailleurs dans le livre de Paraná, déjà cité à la note 2.

4. Pour mieux comprendre la création du PT, je renvoie le lecteur à l'important travail de Garcia (2000).

5. Le frère de Lula, Frei Chico, se plaint de sa timidité quand il était enfant, et ne savait pas « crier » pour vendre ses petites marchandises sur les quais de Santos (Paraná 2008 : 210). Dans ce même livre on apprend que dans l'âge adulte les deux frères ont suivi de brefs cours de Rhétorique.

6. Cet énoncé exclamatif et « euphorisant " peut sembler tendancieux. Il faut donc l'expliquer : le Brésil a été pendant très longtemps victime d'une politique de classe et aussi anti-raciale. Jusqu'à la fin du mandat du président Lula, si on ne pouvait pas affirmer que «tout allait bien dans le meilleurs des mondes" comme le dirait Pangloss (le personnage voltairien) on peut quand même dire que le pays a beaucoup évolué. En plus, il faut convenir que, dans ce contexte, le fait qu'un ouvrier, métis, barbu, qui avait dans sa jeunesse l'air farouche, accidenté de travail (il a perdu un doigt) ait pu se faire élire député dans l'état le plus riche du Brésil (São Paulo) est sans doute surprenant.

7. J'utilise ici sujet-interprétant dans l'acception stricte adoptée par Charaudeau (1983).

8. Pour cet article, on a choisi d'analyser neuf extraits issus de trois discours de Lula : les sept premiers appartiennent à ses discours d'investiture (2003 et 2007) et les deux autres sont issus 
d'un discours de sa première campagne (2002). Mais il faut préciser que Lula a souvent fait intervenir des bribes de son récit de vie dans ses allocutions publiques et cela à un point tel que, lors des dernières campagnes présidentielles au Brésil (2010) quelques candidats ont repris sa façon de parler.

9. En portugais : «é necessário incrementar - e muito - o mercado interno, fortalecendo as pequenas e microempresas. É necessário também investir em capacitação tecnológica e infraestrutura voltada para o escoamento da produção ».

10. En portugais : «Mudança »; esta é a palavra-chave, esta foi a grande mensagem da sociedade brasileira nas eleições de outubro. A esperança finalmente venceu o medo e a sociedade brasileira decidiu que estava na hora de trilhar novos caminhos ".

11. Lula avait un léger zézaiement, qui serait pardonné chez d'autres, mais pas chez un candidat à la présidence du pays.

12. En portugais : «O Brasil é um País imenso, um continente de alta complexidade humana, ecológica e social, com quase 175 milhões de habitantes. Não podemos deixá-lo seguir à deriva, ao sabor dos ventos, carente de um verdadeiro projeto de desenvolvimento nacional [...]. Se queremos transformá-lo, a fim de vivermos em uma Nação em que todos possam andar de cabeça erguida, teremos que exercer quotidianamente duas virtudes: a paciência e a perseverança ».

13. Il faut songer au fait que Lula a été élu, pour son premier mandat, surtout parce qu'il a eu l'adhésion de quelques intellectuels de gauche (entre autres), mais surtout parce qu'il a pu compter sur l'appui des gens très simples, issus des couches vraiment pauvres de la population brésilienne. S'il avait inséré, dans son discours d'investiture, des mots « difficiles ", adressés aux milliers de personnes qui s'aggloméraient pour l'écouter dans les jardins du palais présidentiel, il se serait éloigné non seulement de lui-même mais aussi de la grande masse des Brésiliens qui l'ont fait accéder au pouvoir et qui attendaient du nouveau président des paroles faisant partie de leur univers de gens simples. En ce sens, même les clichés peuvent fonctionner de façon argumentative.

14. En portugais : «Cada um de nós, brasileiros, sabe que o que fizemos até hoje não foi pouco, mas sabe também que podemos fazer muito mais. Quando olho a minha própria vida de retirante nordestino, de menino que vendia amendoim e laranja no cais de Santos, que se tornou torneiro mecânico e líder sindical, que um dia fundou o Partido dos Trabalhadores e acreditou no que estava fazendo, que agora assume o posto de Supremo Mandatário da Nação, vejo e sei, com toda a clareza e com toda a convicção, que nós podemos muito mais ".

15. En portugais : «Quatro anos atrás, nesta Casa, em um primeiro de janeiro, vivi a experiência mais importante de minha vida - a de assumir a presidência do meu país. Não era apenas a realização de um sonho individual. O que então ocorreu foi o resultado de um poderoso movimento histórico do qual eu me sentia - e ainda hoje me sinto - parte e humilde instrumento. Pela primeira vez, um homem nascido na pobreza, que teve que derrotar o risco crônico da morte na infância e vencer, depois, a desesperança na idade adulta chegava, pela disputa democrática, ao mais alto posto da República. Pela primeira vez, a longa jornada de um retirante, que começara, como a de milhões de nordestinos, em cima de um pau-de-arara, terminava, como expressão de um projeto coletivo, na rampa do Planalto ».

16. En portugais : «Minhas Senhoras e meus senhores,//Reconheço que Deus tem sido generoso comigo.//Mais que mereço. //Eu pedi forças... e Deus me deu dificuldades para fazer-me forte.// Eu pedi sabedoria... e Deus me deu problemas para resolver.//Eu pedi prosperidade... e Deus me deu cérebro e músculos para trabalhar.//Eu pedi coragem... e Deus me deu perigos para superar.//Eu pedi amor... e Deus me deu pessoas com dificuldades para ajudar.//Eu pedi dádivas... e Deus me deu oportunidades.//Eu não recebi nada do que pedi, mas eu recebi tudo o que precisava ".

17. En portugais : « eu me lembro da minha mãe que tinha medo que eu entrasse no Sindicato, com medo de eu ser preso. Ela morreu e eu estava preso e ela não sabia que eu estava preso. Essas 
pessoas que morreram e não conseguiram chegar conosco até agora, pode ter certeza, que estão lá em cima olhando prá nós, rindo de alegria, porque nos conseguimos construir o sonho de algumas gerações ».

18. En portugais: «E eu quero que vocês saibam que minha mãe dizia [silêncio] minha mãe nasceu e morreu analfabeta e a minha mãe dizia "meu filho, a única coisa que o homem não pode perder é o direito de andar de cabeça erguida e olhar nos olhos das pessoas com quem está conversando ».

19. Il est courant au Brésil, dans les familles vraiment très pauvres, que les hommes s'en aillent et laissent les enfants au soin de la mère.

\section{RÉSUMÉS}

Cet article propose une réflexion sur le rôle du récit de vie dans les discours politiques, et plus précisément dans les discours d'investiture à la présidence du Brésil de Luis Ignacio Lula da Silva. Il analyse quelques fragments qui mêlent expressément des souvenirs de sa vie passée aux discours politiques d'actualité, mais aussi qui font allusion à sa vie de façon indirecte. Dans le premier discours, Lula se présente comme un personnage de l'histoire, un «il» qui veut travailler pour la collectivité; néanmoins, les allusions à sa trajectoire de vie le légitiment comme celui qui peut promouvoir le changement requis par le peuple. Dans le second discours, Lula utilise depuis le début un «je» ancré dans sa propre histoire: sa "petite " histoire personnelle semble ainsi s'incorporer à la « grande » histoire d'une nation. En ce sens, le récit de vie comprend une dimension argumentative qui permet de construire un ethos présidentiel par le jeu qu'il instaure entre l'individuel et le collectif, créant ainsi un effet de pathos qui appelle à l'identification. On s'interroge dans ce cadre sur la question du populisme dans son rapport aux stratégies de captation propres au discours politique.

This paper analyzes fragments of President Luiz Inácio Lula da Silva's inaugural speeches. Lula da Silva served as the President of Brazil for two consecutive terms in office. In his first inaugural speech, Lula da Silva refers to himself as a historical character, and uses the pronoun "he" (or "not-I"), a "he" at the service of the community. However, his path in life, mentioned in a single paragraph, legitimizes him as someone who is able to bring forward the changes demanded by the people. From the beginning of his second inaugural speech, Lula da Silva uses the pronoun "I", which points to his own story: therefore, his "small, personal story" seems to be embedded into the "great History" of a nation. Can life narratives function as argumentative strategies? Or, in this particular case, is the retelling of Lula da Silva's life story in his speech simply part of a truly passionate and emotional address, delivered by a man of the people, who won the presidency of his country?

\section{INDEX}

Mots-clés : discours d'inauguration, discours politique, Lula (Luis Ignacio Lula da Silva), populisme, récit de vie, stratégie argumentative

Keywords : argumentative strategies, inaugural address, life story, Lula (Luis Ignacio Lula da Silva), political discourse, populism 
AUTEUR

IDA LUCIA MACHADO

Universidade Federal de Minas Gerais 